\title{
Which patients are not included in the English Cancer Waiting Times monitoring dataset, 2009-2013? Implications for use of the data in research
}

C Di Girolamo ${ }^{1,2}$, S Walters ${ }^{1}$, C Gildea ${ }^{3}$, S Benitez Majano ${ }^{1}$, M P Coleman ${ }^{1}$, B Rachet ${ }^{1}$ and M Morris ${ }^{\star}, 1$

${ }^{1}$ Cancer Survival Group, Faculty of Epidemiology and Population Health, Department of Non-Communicable Disease Epidemiology, London School of Hygiene \& Tropical Medicine, Keppel Street, London WC1E 7HT, UK; ${ }^{2}$ Department of Medical and Surgical Sciences, Alma Mater Studiorum, University of Bologna, Via Zamboni, 33, 40126 Bologna, Italy and ${ }^{3}$ National Cancer Registration and Analysis Service, Public Health England, Vulcan House Steel, 6 Millsands, Sheffield S3 8NU, UK

Background: Cancer waiting time targets are routinely monitored in England, but the Cancer Waiting Times monitoring dataset (CWT) does not include all eligible patients, introducing scope for bias.

Methods: Data from adults diagnosed in England (2009-2013) with colorectal, lung, or ovarian cancer were linked from CWT to cancer registry, mortality, and Hospital Episode Statistics data. We present demographic characteristics and net survival for patients who were and were not included in CWT.

Results: A CWT record was found for $82 \%$ of colorectal, $76 \%$ of lung, and $77 \%$ of ovarian cancer patients. Patients not recorded in CWT were more likely to be in the youngest or oldest age groups, have more comorbidities, have been diagnosed through emergency presentation, have late or missing stage, and have much poorer survival.

Conclusions: Researchers and policy-makers should be aware of the limitations in the completeness and representativeness of CWT, and draw conclusions with appropriate caution.

Successive national cancer plans and strategies for England have included targets to reduce waiting times to diagnosis and treatment for all cancers (Department of Health, 2000, 2007, 2011; Independent Cancer Taskforce, 2015). These include a maximum 2-week wait (TWW) between an urgent referral from a general practitioner (GP) to being seen by a specialist, a maximum 62 days from the GP's urgent referral to the start of first treatment, and a maximum 31 days from the decision to treat a patient to the start of treatment. A new 28-day target to confirm or exclude a cancer diagnosis has also been proposed (Independent Cancer Taskforce, 2015).

Waiting time targets are considered to be important indicators of the quality of cancer care. National cancer waiting time statistics have been published quarterly by NHS England since $2013-2014$ (Cancer Waiting Times Team, 2016) and previously by the Department of Health. Performance varies widely across the country: many Clinical Commissioning Groups fall below current operational standards, and adherence to the 62-day target has been decreasing since 2014 (Cancer Waiting Times Team, 2016).

The English National Cancer Waiting Times monitoring dataset (CWT), is the basis for these official statistics. The data are collected by 'the provider that is commissioned to deliver the activity' (Cancer Waiting Times Team, 2015). CWT only contains diagnosis and treatment information on cancer patients who were offered treatment within the NHS, including those who refused treatment or were assigned to active monitoring (Cancer Waiting 
Times Team, 2015), whichever diagnosis route they came through. However, not all eligible cancer patients are included and the extent of incompleteness is unclear. We examine recent CWT data for three cancers, linked to individual cancer patient data, to compare the characteristics of patients who were and were not included in CWT.

\section{MATERIALS AND METHODS}

Data sources and study population. All adults (15 - 99 years) who were diagnosed in England during 2009-2013 with colorectal, non-small cell lung or ovarian cancer were included.

CWT diagnosis and treatment data were linked at individual level with the national cancer registry data (including vital status at 31 December 2014), the Hospital Episode Statistics data, and the Routes to Diagnosis dataset (Elliss-Brookes et al, 2012). Demographic information included age, sex, and deprivation quintile. Stage at diagnosis and Charlson Comorbidity Index score (Charlson et al, 1987) were derived using algorithms applied to these datasets and audit data, where available (Benitez-Majano et al, 2016; Maringe et al, 2017).

Statistical analysis. We examined the sociodemographic and clinical characteristics of cancer patients and estimated net survival at 1 year. We report results by patient age group, deprivation quintile, and tumour stage. Net survival can be interpreted as survival from the cancer, accounting for the mortality from other causes, using life tables of the England general population stratified by age, sex, calendar year, and region (Spika, 2015).

\section{RESULTS}

During 2009-2013, 164890 colorectal, 171208 non-small cell lung, and 24545 ovarian cancer patients were registered in England, of whom $82 \%, 76 \%$, and $77 \%$, respectively, were included in CWT for first and/or subsequent treatments (Table 1).

The completeness of CWT improved slightly during $2009-2011$, more for lung and ovarian cancers than for colorectal cancer, then plateaued until 2013. The percentage of patients included varied by patient and tumour factors, with nearly all differences statistically significant in a $\chi^{2}$-test at $P<0.001$ (Table 1 , Figure 1).

There was a strong J-shaped age pattern in the probability of inclusion in CWT (Figure 1): the youngest and, especially, the oldest patients were least likely to be included. Among those older than 70 years, over a quarter with colorectal cancer and around $40 \%$ with lung or ovary cancer had no record in CWT. More affluent patients were less likely to have a CWT record. Women with colorectal cancer were slightly less likely to have a CWT record than men (80.2 vs $82.7 \%$ ), but there was no evidence of a difference between the sexes for lung cancer $(P=0.211)$.

A CWT record was missing for more than half of patients whose route to diagnosis was unknown, and for around a third of those diagnosed through an emergency presentation.

More than $85 \%$ of patients with stage I and II tumours were recorded in CWT (Figure 1). Among colorectal and lung cancer patients, those with missing stage were also the most likely to be missing from CWT, although the proportion was similar to patients diagnosed at stage IV. Among women with ovarian cancer,

Table 1. Presence of a record in the CWT by selected characteristics of patients

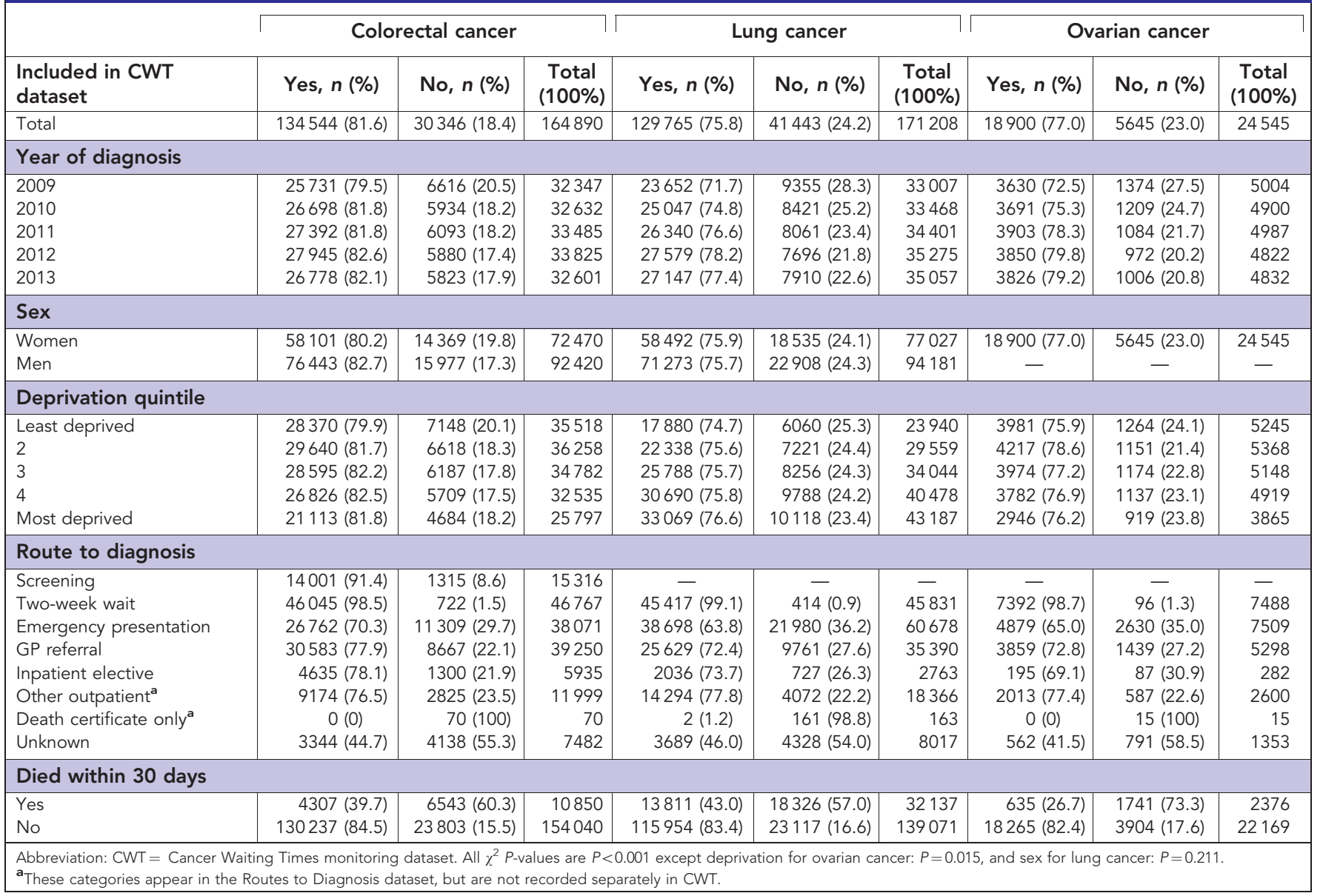



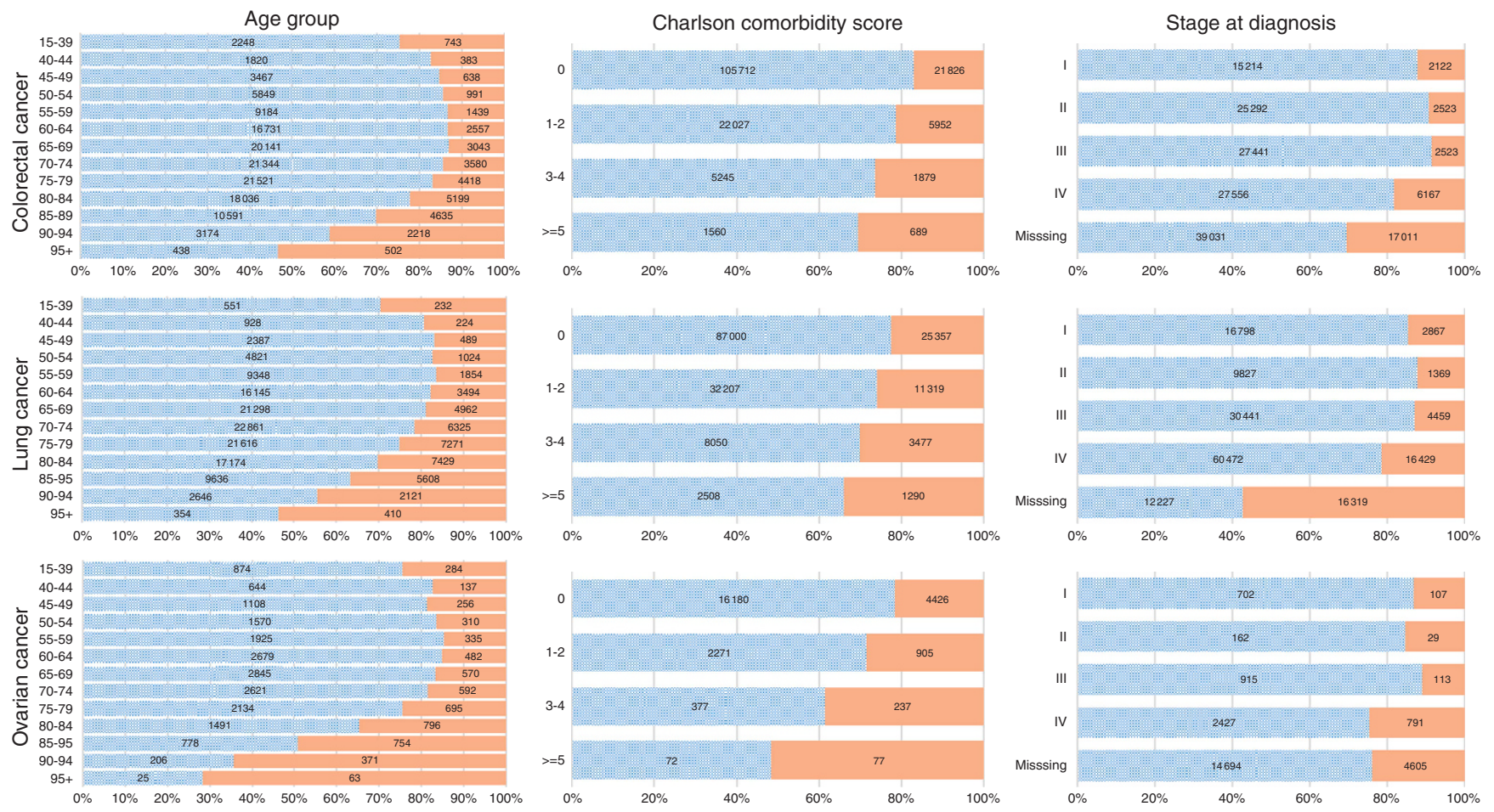

With CWT info \% Without CWT info \%

Figure 1. Age, level of comorbidity, and stage at diagnosis of colorectal, lung, and ovarian cancer patients by presence of a matching record in the Cancer Waiting Times monitoring dataset.

a similar proportion of cases with stage IV and missing stage tumours were not recorded (24\%).

Patients with more comorbidity were less likely to be recorded in CWT (Figure 1). For women with ovarian cancer, those with no comorbidities were $30 \%$ more likely to have a CWT record than those with the most comorbidity.

Patients who died within 30 days of diagnosis were much less likely to have a CWT record, ranging from $60 \%$ (colorectal and lung cancers) to $73 \%$ (ovarian cancer), but $15-18 \%$ of those who survived at least 30 days were also not captured (Table 1), with a similar J-shaped age pattern to that of the whole cohort.

For all three cancers, 1-year net survival was far lower among patients who were not captured by CWT than among those who were (colorectal cancer: $52.2 \%$ (95\% CI, $51.6-52.8 \%$ ) compared to 81.4\% (81.2 - 81.7\%); lung cancer: $17.0 \%$ (16.6 - 17.4\%) compared to $39.0 \%(38.7-39.2 \%)$; ovarian cancer: $40.4 \%(39.1-41.7 \%)$ compared to $77.1 \%(76.4-77.7 \%)$; Table 2$)$. The differences generally increased with increasing age, stage, and deprivation. The differences were particularly stark for those with stage IV disease. There was very little difference in survival, among the youngest patients (aged $15-44$ years), between those who did and did not appear in CWT.

\section{DISCUSSION}

Using a new approach to examine the English CWT, we linked individual data from several sources to describe the characteristics and short-term survival of patients who were not included in the dataset. Around one-fifth of patients diagnosed with colorectal, lung, or ovarian cancer during 2009-2013 did not have a CWT record. Proportions were highest among elderly patients and those with comorbid conditions, mirroring patterns for those with missing stage information (Adams et al, 2004; Worthington et al, 2008). Patients missing from CWT were also more likely to have advanced disease or missing stage information: these factors are highly correlated (Barclay et al, 2016). However, more than a quarter of the youngest patients and more than $10 \%$ of those with early-stage disease also lacked a CWT record, suggesting that the recording of CWT data could be improved.

Several mechanisms may help explain the pattern and extent of missing records. Some treatments may not be well recorded (e.g., pain relief or transfusions). The CWT does not include data on patients who died before treatment could commence, even if a decision to treat had been made. However, in our data, only $22 \%$, $44 \%$, and $31 \%$ of colorectal, lung, and ovarian cancer patients without a CWT record, respectively, died within 30 days of diagnosis. Services not commissioned by the NHS are also beyond the scope of CWT data collection, so patients treated in the private sector, including palliative care in non-NHS organisations, are not captured (Cancer Waiting Times Team, 2015). The extent of this is unclear, but it is reported that around $11 \%$ of the UK population has some private health insurance (The King's Fund, 2014). The proportion of colorectal and ovarian cancer patients included in CWT was indeed lower among those in the most affluent quintile, who are more likely to seek private care.

A CWT record may be missing because of a clinical decision not to treat a patient: this may explain why older and sicker patients are less likely to be included. Indeed, under- or sub-optimal treatment in the elderly has been reported in England (National Cancer Equality Initiative, 2012; Forrest et al, 2014; Lawler et al, 2014).

One-year survival was generally much lower among patients with no CWT record, but no survival differences were found among the youngest patients between those with and without a record. This suggests that younger patients may well have had treatment that was not captured in CWT.

There may be some administrative under-reporting by which patients received treatment that was not recorded in CWT. The presence in CWT records of patients who received 'subsequent treatment' without any record of first treatment (5-9\% depending 
Table 2. One-year NS and presence of CWT record by age, stage, and deprivation of patients

\begin{tabular}{|c|c|c|c|c|c|c|}
\hline & \multicolumn{2}{|c|}{ Colorectal cancer } & \multicolumn{2}{|c|}{ Lung cancer } & \multicolumn{2}{|c|}{ Ovarian cancer } \\
\hline & Yes & No & Yes & No & Yes & No \\
\hline $\begin{array}{l}\text { Included in CWT } \\
\text { dataset }\end{array}$ & NS \% $(95 \% \mathrm{Cl})$ & NS \% $(95 \% \mathrm{Cl})$ & NS \% $(95 \% \mathrm{Cl})$ & NS \% $(95 \% \mathrm{Cl})$ & NS \% $(95 \% \mathrm{Cl})$ & NS \% $(95 \% \mathrm{Cl})$ \\
\hline Overall & $81.4(81.2-81.7)$ & $52.2(51.6-52.8)$ & $39.0(38.7-39.2)$ & $17.0(16.6-17.4)$ & $77.1(76.4-77.7)$ & $40.4(39.1-41.7)$ \\
\hline \multicolumn{7}{|l|}{ Age groups, years } \\
\hline $15-44$ & $86.9(85.8-87.9)$ & $87.9(86.0-89.8)$ & $52.0(49.4-54.5)$ & $56.6(52.0-61.1)$ & $90.7(89.2-92.2)$ & $88.9(85.9-91.9)$ \\
\hline $45-54$ & $87.7(87.0-88.4)$ & $77.8(75.8-82.9)$ & $46.0(44.9-47.2)$ & $29.8(27.5-32.1)$ & $89.4(88.2-90.5)$ & $79.0(75.6-82.3)$ \\
\hline $55-64$ & $87.7(87.3-88.1)$ & 71.8 (70.4-73.2) & $44.0(43.4-44.6)$ & $22.2(21.1-23.3)$ & $84.1(83.1-85.2)$ & $59.8(56.4-63.2)$ \\
\hline $65-74$ & $85.2(84.8-85.6)$ & $61.2(60.0-62.4)$ & $42.1(41.6-42.6)$ & $18.1(17.4-18.8)$ & 78.0 (76.9-79.2) & $42.8(39.9-45.7)$ \\
\hline $75+$ & $74.0(73.6-74.4)$ & $39.2(38.4-40.0)$ & $32.4(31.9-32.8)$ & $13.6(13.2-14.1)$ & $57.3(55.8-58.8)$ & $17.6(16.1-19.1)$ \\
\hline \multicolumn{7}{|l|}{ Stage } \\
\hline I & $98.4(98.1-98.8)$ & 94.0 (92.7-95.3) & $85.2(84.6-85.8)$ & $69.9(68.1-71.7)$ & $97.4(96.8-98.1)$ & 95.6 (93.7-97.5) \\
\hline II & $94.6(94.3-95.0)$ & $82.7(81.1-84.4)$ & $69.0(68.0-69.9)$ & $45.1(42.3-47.8)$ & $91.0(89.1-92.9)$ & $73.9(66.6-81.1)$ \\
\hline III & $89.8(89.3-90.2)$ & $71.1(69.2-73.0)$ & $44.5(44.0-45.1)$ & $19.8(18.6-21.0)$ & $80.1(78.9-81.2)$ & $47.4(43.6-51.2)$ \\
\hline IV & $52.2(51.6-52.8)$ & $21.4(20.4-22.5)$ & $19.2(18.9-19.5)$ & $7.0(6.6-7.4)$ & $62.8(61.2-64.5)$ & $20.8(18.2-23.4)$ \\
\hline Missing & $81.0(80.6-81.4)$ & $50.8(50.0-51.5)$ & $35.1(34.3-36.0)$ & $14.7(14.2-15.3)$ & $68.2(67.0-69.5)$ & $33.4(31.8-35.1)$ \\
\hline \multicolumn{7}{|c|}{ Deprivation quintile } \\
\hline Least deprived & $83.5(83.1-84.0)$ & $61.5(60.3-62.7)$ & $39.9(39.2-40.6)$ & $22.2(21.2-23.3)$ & $78.7(77.4-80.1)$ & $49.4(46.6-52.2)$ \\
\hline 2 & $82.6(82.1-83.1)$ & $55.4(54.2-56.7)$ & $39.3(38.6-39.9)$ & $18.4(17.5-19.3)$ & $76.1(74.7-77.4)$ & $44.5(41.6-47.4)$ \\
\hline 3 & $81.3(80.8-81.8)$ & $50.9(49.6-52.2)$ & $38.6(38.0-39.2)$ & $16.5(15.7-17.3)$ & 77.1 (75.7-78.4) & $37.8(35.0-40.6)$ \\
\hline 4 & $79.9(79.4-80.5)$ & $46.3(44.9-47.7)$ & $38.4(37.8-38.9)$ & $15.9(15.1-16.6)$ & $75.9(74.4-77.3)$ & $33.9(31.1-36.8)$ \\
\hline Most deprived & 78.9 (78.3-79.5) & $42.2(40.7-43.7)$ & $39.0(38.5-39.6)$ & $14.5(13.8-15.2)$ & $77.8(76.2-79.4)$ & $33.9(30.8-37.1)$ \\
\hline
\end{tabular}

Abbreviations: $\mathrm{Cl}=$ confidence interval; $\mathrm{CWT}=$ Cancer Waiting Times monitoring dataset; NS = net survival. Bold font indicates that Cls in the 'No' column do not overlap with those in the corresponding 'Yes' column.

on the cancer) lends weight to this. The proportion of patients with no CWT record was also higher among those with missing information on stage and with an unknown route to diagnosis, suggesting that there is a group of patients whose information is generally poorly captured, due to shortcomings in data transmission system or clinical documentation.

Our novel approach offers a clear picture of the characteristics of patients who are not included in CWT, but it cannot fully illuminate the mechanisms of this incompleteness. The extent to which the missing data create scope for selection bias must be considered, especially if the reason that patients are not included is related to whether they meet the waiting times target. The generalisability of results from CWT to the whole population of cancer patients recorded in the cancer registration data may be limited, because they do not reflect the outcomes for patients who were treated outside the NHS, or of patients who were not captured despite having received some treatment. These sources of bias should be considered when interpreting the results or using the CWT data to evaluate cancer outcomes at patient level.

The CWT dataset is an important source of data: it allows monitoring of key indicators of NHS performance in cancer care and the targets encourage timely treatment. However, researchers and policy-makers should be aware of these limitations in the completeness and representativeness of the CWT data, and should draw any conclusions with appropriate caution.

\section{ACKNOWLEDGEMENTS}

The Cancer Waiting Times monitoring dataset was provided by NHS England through Public Health England. We are grateful to the members of the CRUK EDAG Scientific Advisory Group for their help in developing this project. We thank members of the Cancer Survival Group at LSHTM for their advice and support. CDG, SW, SBM, and MM are funded by the Cancer Research UK Early Diagnosis Commissioned Policy Research Programme at the London School of Hygiene and Tropical Medicine (award number
C7923/A18348). The funding body collaborated in the design of the study but had no role in the collection and analysis of data, interpretation of results, or in writing the manuscript. The Cancer Survival Group obtained ethical and statutory approvals to use these routinely collected data from the National Research Ethics Service Committee London-Camden \& Islington on 28 May 2013 (Research Ethics Committee reference 13/LO/0610, confirmed on 29 January 2015). All the data are anonymised and the researchers had no access to personally identifiable data.

\section{CONFLICT OF INTEREST}

The authors declare no conflict of interest.

\section{REFERENCES}

Adams J, Audisio RA, White M, Forman D (2004) Age-related variations in progression of cancer at diagnosis and completeness of cancer registry data. Surg Oncol 13(4): 175-179.

Barclay M, Gildea C, Poole J, Hirschowitz L, Menon U, Nordin A (2016) Factors affecting short-term mortality in women with ovarian, tubal, or primary peritoneal cancer: population-based cohort analysis of English National Cancer registration data. Int J Gynecol Cancer 26(1): $56-65$.

Benitez-Majano S, Fowler H, Maringe C, Di Girolamo C, Rachet B (2016) Deriving stage at diagnosis from multiple population-based sources: colorectal and lung cancer in England. Br J Cancer 115(3): 391-400.

Cancer Waiting Times Team (2015) National Cancer Waiting Times Monitoring Dataset Guidance, version 9.0 NHS England: London.

Cancer Waiting Times Team (2016) Cancer waiting times-Commissioner Time Series by CCG Q1 2013-2014 to Q1 2016-2017. NHS England: London; Available at: https://www.england.nhs.uk/statistics/statisticalwork-areas/cancer-waiting-times/.

Charlson ME, Pompei P, Ales KL, MacKenzie CR (1987) A new method of classifying prognostic comorbidity in longitudinal studies: development and validation. J Chronic Dis 40(5): 373-383. 
Department of Health (2000) The NHS Cancer Plan: a Plan for Investment, a Plan for Reform. Department of Health: London.

Department of Health (2007) Cancer Reform Strategy. Department of Health: London.

Department of Health (2011) Improving Outcomes: a Strategy for Cancer. Department of Health: London.

Elliss-Brookes L, McPhail S, Ives A, Greenslade M, Shelton J, Hiom S, Richards M (2012) Routes to diagnosis for cancer-determining the patient journey using multiple routine datasets. Br J Cancer 107(8): 1220-1226.

Forrest LF, Adams J, White M, Rubin G (2014) Factors associated with timeliness of post-primary care referral, diagnosis and treatment for lung cancer: population-based, data-linkage study. Br J Cancer 111(9): 1843-1851.

Independent Cancer Taskforce (2015) Achieving World-class Cancer Outcomes: a Strategy for England 2015-2020. NHS England: London.

Lawler M, Selby P, Aapro M, Duffy S (2014) Ageism in cancer care. BMJ 348: g1614.

Maringe C, Fowler H, Rachet B, Luque-Fernández MA (2017) Reproducibility, reliability and validity of population-based administrative health data for the assessment of cancer non-related comorbidities. PLoS One 12(3): e0172814.

National Cancer Equality Initiative (2012) The Impact of Patient Age on Clinical Decision-Making In Oncology. Department of Health.

Spika D (2015) Cancer Survival Group UK life tables, London. Available at: http://csg.lshtm.ac.uk/tools-analysis/uk-life-tables/.

The King's Fund (2014) The UK Private Health Market. The King's Fund: London.

Worthington JL, Koroukian SM, Cooper GS (2008) Examining the characteristics of unstaged colon and rectal cancer cases. Cancer Detect Prev 32(3): 251-258.

(c) (i) This work is licensed under the Creative Commons Attribution 4.0 International License. To view a copy of this license, visit http://creativecommons.org/licenses/by/4.0/

(C) The Author(s) named above 2018 